\title{
Unlocking a signal of introgression from codons in Lachancea kluyveri using a mutation-selection model
}

\author{
Cedric Landerer ${ }^{1,2,3^{*}}$ (D), Brian C. O'Meara ${ }^{1,2}$, Russell Zaretzki ${ }^{2,4}$ and Michael A. Gilchrist ${ }^{1,2}$
}

\begin{abstract}
Background: For decades, codon usage has been used as a measure of adaptation for translational efficiency and translation accuracy of a gene's coding sequence. These patterns of codon usage reflect both the selective and mutational environment in which the coding sequences evolved. Over this same period, gene transfer between lineages has become widely recognized as an important biological phenomenon. Nevertheless, most studies of codon usage implicitly assume that all genes within a genome evolved under the same selective and mutational environment, an assumption violated when introgression occurs. In order to better understand the effects of introgression on codon usage patterns and vice versa, we examine the patterns of codon usage in Lachancea kluyveri, a yeast which has experienced a large introgression. We quantify the effects of mutation bias and selection for translation efficiency on the codon usage pattern of the endogenous and introgressed exogenous genes using a Bayesian mixture model, ROC SEMPPR, which is built on mechanistic assumptions about protein synthesis and grounded in population genetics.
\end{abstract}

Results: We find substantial differences in codon usage between the endogenous and exogenous genes, and show that these differences can be largely attributed to differences in mutation bias favoring $A / T$ ending codons in the endogenous genes while favoring $\mathrm{C} / \mathrm{G}$ ending codons in the exogenous genes. Recognizing the two different signatures of mutation bias and selection improves our ability to predict protein synthesis rate by $42 \%$ and allowed us to accurately assess the decaying signal of endogenous codon mutation and preferences. In addition, using our estimates of mutation bias and selection, we identify Eremothecium gossypii as the closest relative to the exogenous genes, providing an alternative hypothesis about the origin of the exogenous genes, estimate that the introgression occurred $\sim 6 \times 10^{8}$ generation ago, and estimate its historic and current selection against mismatched codon usage.

Conclusions: Our work illustrates how mechanistic, population genetic models like ROC SEMPPR can separate the effects of mutation and selection on codon usage and provide quantitative estimates from sequence data.

Keywords: Codon usage, Population genetics, Introgression, Mutation, Selection

${ }^{*}$ Correspondence: landerer@mpi-cbg.de

'Department of Ecology \& Evolutionary Biology, University of Tennessee,

37996 Knoxville, TN, USA

${ }^{2}$ National Institute for Mathematical and Biological Synthesis, 37996 Knoxville,

TN, USA

Full list of author information is available at the end of the article

(C) The Author(s). 2020 Open Access This article is licensed under a Creative Commons Attribution 4.0 International License, which permits use, sharing, adaptation, distribution and reproduction in any medium or format, as long as you give appropriate credit to the original author(s) and the source, provide a link to the Creative Commons licence, and indicate if changes were made. The images or other third party material in this article are included in the article's Creative Commons licence, unless indicated otherwise in a credit line to the material. If material is not included in the article's Creative Commons licence and your intended use is not permitted by statutory regulation or exceeds the permitted use, you will need to obtain permission directly from the copyright holder. To view a copy of this licence, visit http://creativecommons.org/licenses/by/4.0/. The Creative Commons Public Domain Dedication waiver (http://creativecommons.org/publicdomain/zero/1.0/) applies to the data made available in this article, unless otherwise stated in a credit line to the data. 


\section{Background}

Synonymous codon usage patterns varies within a genome and between taxa, reflecting differences in mutation bias, selection, and genetic drift. The signature of mutation bias is largely determined by the organism's internal or cellular environment, such as their DNA repair genes or UV exposure. While this mutation bias is an omnipresent evolutionary force, its impact can be obscured or amplified by selection. The signature of selection on codon usage is largely determined by an organism's cellular environment alone, such as, but not limited to, its tRNA species, their copy number, and their post-transcriptional modifications. In general, the strength of selection on codon usage is assumed to increase with its expression level [1-3], specifically its protein synthesis rate [4]. Thus as protein synthesis increases, codon usage shifts from a process dominated by mutation to a process dominated by selection. The overall efficacy of mutation and selection on codon usage is a function of the organism's effective population size $N_{e}$. ROC SEMPPR allows us to disentangle the evolutionary forces responsible for the patterns of codon usage bias [5-7] (CUB) encoded in an species' genome, by explicitly modeling the combined evolutionary forces of mutation, selection, and drift [4, 8-10]. In turn, these evolutionary parameters should provide biologically meaningful information about the lineage's historical cellular and external environment.

Most studies implicitly assume that the CUB of a genome is shaped by a single cellular and external environment. However, this assumption is clearly violated to increasing degrees via horizontally gene transfer, large scale introgressions, and hybrid species formation. In these scenarios, one would expect to see the signature of multiple cellular environments in a genome's CUB [11, 12]. Indeed, differences in CUB between linages have been proposed to have a major effect on their rates of gene transfer with rates declining with differences in their CUB. On a more practical level, if differences in codon usage of transferred genes are not taken into account for, they may distort the interpretation of codon usage patterns. Such distortion could lead to the wrong inference of codon preference for an amino acid $[8,10]$, underestimate the variation in protein synthesis rate, or distort estimates of mutation bias when analyzing a genome.

To illustrate these ideas, we analyze the CUB of the genome of the yeast Lachancea kluyveri using ROC SEMPPR, a population genetics based model of synonymous codon usage evolution that accounts for and, in turn, can estimate the contribution of mutation bias $\Delta M$, selection bias. The mathematics of ROC SEMPPR are derived on a mechanistic description of ribosome movement along an mRNA, although the approximation of other biological mechanisms could also be consistent with the model. Broadly speaking, ROC SEMPPR allows us to quantify the cellular environment in which genes have evolved by separately estimating the effects of mutation bias and selection bias on codon usage $\Delta \eta$ between synonymous codons and protein synthesis rate $\phi$ to the patterns of codon usage observed within a set of genes. Briefly, the set of $\Delta M$ for an amino acid quantifies the relative differences in mutational stability or bias between the synonymous codons of the amino acid $\mathbb{S}$. In the absence of selection bias (or equivalently when gene expression $\phi=0$ ), the equilibrium frequency of synonymous codon $i$ is simply $\exp \left[-\Delta M_{i}\right] /\left(\sum_{j \in \mathbb{S}} \exp \left[-\Delta M_{j}\right]\right)$. Because the time units of protein production rate have no intrinsic time scale, we define the average protein production rate for a set of genes to be one, i.e. $\bar{\phi}=1$ by definition [10]. In order to facilitate comparisons between gene sets, we express both, $\Delta M$ and $\Delta \eta$, as deviation from the mean of each synonymous codon family (see Methods for details). Nevertheless, the difference $\Delta \eta$ describes the difference in fitness between two synonymous codons relative to drift for a gene whose protein production rate $\phi$ is equal to the average rate of protein production $\bar{\phi}$ across the set of genes. In other words, for a gene whose protein is expressed at the average rate, for any two given synonymous codons $i$ and $j, \Delta \eta_{i}-\Delta \eta_{j}=N_{e} s$.

The Lachancea clade diverged from the Saccharomyces clade, prior to its whole genome duplication $\sim 100$ Mya ago $[13,14]$. Since that time, L. kluyveri, which is sister species to all other Lachancea spp., has experienced a large introgression of exogenous genes ( $1 \mathrm{Mb}, 457$ genes) which is found in all of its populations $[15,16]$, but in no other known Lachancea species [17]. The introgression replaced the left arm of the $\mathrm{C}$ chromosome and displays a $13 \%$ higher GC content than the endogenous L. kluyveri genome $[15,16]$. Previous studies suggest that the source of the introgression is probably a currently unknown or potentially extinct Lachancea lineage based on gene concatenation or synteny relationships [15-18]. These characteristics make L. kluyveri an ideal model to study the effects of an introgressed cellular environment and the resulting mismatch in codon usage.

While previous studies [8, 9] have used information on gene expression to separate the effects of mutation and selection on codon usage, ROC SEMPPR does not need such information but can provide it. ROC SEMPPR's resulting predictions of protein synthesis rates have been shown to be on par with laboratory measurements $[8,10]$. In contrast to often used heuristic approaches to study codon usage $[5,6,19]$, ROC SEMPPR explicitly incorporates and distinguishes between mutation and selection effects on codon usage and properly weights its estimates by amino acid usage [20]. We use ROC SEMPPR to separately describe the two cellular environments reflected in the L. kluyveri genome; the signature 
of the endogenous environment reflected in the larger set of non-introgressed genes and the decaying signature of the ancestral, exogenous environment in the smaller set of introgressed genes. Our results indicate that the current difference in GC content between endogenous and exogenous genes is mostly due to the differences in mutation bias $\Delta M$ of their respective cellular environments. Taking the different signatures of $\Delta M$ and selection bias $\Delta \eta$ of the endogenous and exogenous sets of genes substantially improves our ability to predict present day protein synthesis rates $\phi$. These endogenous and exogenous gene set specific estimates of $\Delta M$ and $\Delta \eta$, in turn, allow us to address more refined biological questions. For example, we find support for an alternative origin of the exogenous genes and identify E. gossypii as the nearest sampled relative of the source of the introgressed genes out of the 332 budding yeast lineages with sequenced genomes [21]. While this inference is in contrast to previous work [15-18], we find additional phylogenetic support for via gene tree reconstruction and gene synteny. We also estimate the age of the introgression to be on the order of 0.2 - 1.7 Mya, estimate the selection against these genes, both at the time of introgression and now, and predict a detectable signature of CUB to persist in the introgressed genes for another $0.3-2.8 \mathrm{Mya}$, highlighting the sensitivity of our approach.

\section{Results}

\section{The signatures of two cellular environments within $L$.}

\section{kluyveri's genome}

We used our software package AnaCoDa [22] to compare model fits of ROC SEMPPR to the entire L. kluyveri genome and its genome partitioned into two sets of 4,864 endogenous and 497 exogenous genes. These two set where initially identified based on their striking difference in GC content [15], with very little overlap in GC content between the two sets (Additional file 1: Figure S1a). ROC SEMPPR is a statistical model that relates the effects of mutation bias $\Delta M$, selection bias $\Delta \eta$ between synonymous codons and protein synthesis rate $\phi$, to explain the observed codon usage patterns. Thus, the probability of observing a synonymous codon is proportional to $p \propto$ $\exp (-\Delta M-\Delta \eta \phi)$ [10]. Briefly, $\Delta M$ describes the mutation bias between two synonymous codons at stationarity under a time reversible mutation model. Because ROC SEMPPR only considers the stationary probabilities, only variation in mutation bias, not absolute mutation rates can be detected. $\Delta \eta$ describes the fitness difference between two synonymous codons relative to drift [10]. Since $\Delta \eta$ is scaled by protein synthesis rate $\phi$, this term is dominant in highly expressed genes and tends towards 0 in low expression genes, allowing us to separate the effect of mutation bias and selection bias on codon usage. We express both, $\Delta M$ and $\Delta \eta$, as deviation from the mean of each synonymous codon family which prevents that the choice of the reference codon affects our results (see Methods for details).

Bayes factor strongly support the hypothesis that the $L$. kluyveri genome consists of genes with two different and distinct patterns of codon usage bias rather than a single $(K=\exp (42,294)$; Table 1 , Additional file 1: Figure S2). We find additional support for this hypothesis when we compare our predictions of protein synthesis rate to empirically observed mRNA expression values as a proxy for protein synthesis. Specifically, we improve the variance explained by our predicted protein synthesis rates by $\sim 42 \%$, from $R^{2}=0.33\left(p<10^{10}\right)$ to $0.46\left(p<10^{10}\right)$ (Fig. 1). While the implicit consideration of GC content in this analysis certainly plays a roll, it does not explain the improvement in $R^{2}$ (Additional file 1: Figure S1b).

\section{Comparing differences in the endogenous and exogenous codon usage}

Because ROC SEMPPR defines $\bar{\phi}=1$, it makes the interpretation of $\Delta \eta$ as selection on codon usage of the average gene with $\phi=1$ straightforward and gives us the ability to compare the efficacy of selection $s N_{e}$ across genomes. While it may be expected for the endogenous and exogenous genes to differ in the their codon usage pattern due to the large difference in GC content it is not clear how much of this difference is due to differences in the mutation bias $\Delta M$ or selection bias $\Delta \eta$ between the gene sets. To better understand the differences in the endogenous and exogenous cellular environments, we compared our parameter estimates of $\Delta M$ and $\Delta \eta$ for the two sets of genes. Our estimates of $\Delta M$ for the endogenous and exogenous genes were negatively correlated $(\rho=-0.49$, $p=3.56 \times 10^{-5}$ ), indicating weak similarity with only $\sim 5 \%$ of the codons share the same sign between the two mutation environments (Fig. 2a). Overall, the endogenous genes only show a selection preference for $\mathrm{C}$ and $\mathrm{G}$ ending codons in $\sim 58 \%$ of the codon families. In contrast, the exogenous genes display a strong preference for $\mathrm{A}$ and T ending codons in $\sim 89 \%$ of the codon families.

For example, the endogenous genes show a mutational bias for $\mathrm{A}$ and $\mathrm{T}$ ending codons in $~ 95 \%$ of the codon

Table 1 Model selection of the two competing hypothesis

\begin{tabular}{llllll}
\hline Hypothesis & $\log (\mathcal{L})$ & $n$ & $\log \left(\mathcal{L}_{M}\right)$ & $\log (K)$ & $p$ \\
\hline Combined & $-2,650,047$ & 5,483 & $-2,657,582$ & - & - \\
Separated & $-2,612,397$ & 5,402 & $-2,615,288$ & 42,294 & 0 \\
\hline Combined: mutation bias and selection bias for synonymous codons is shared \\
between endogenous and exogenous genes. Separated: mutation bias and \\
selection bias for synonymous codons is allowed to vary between endogenous and \\
exogenous genes. Reported are the log-likelihood, $\log (\mathcal{L})$, the number of \\
parameters estimated $n$, the log-marginal likelihood $\log \left(\mathcal{L}_{M}\right)$, Bayes Factor K, and \\
the $p$-value of the likelihood ratio test
\end{tabular}




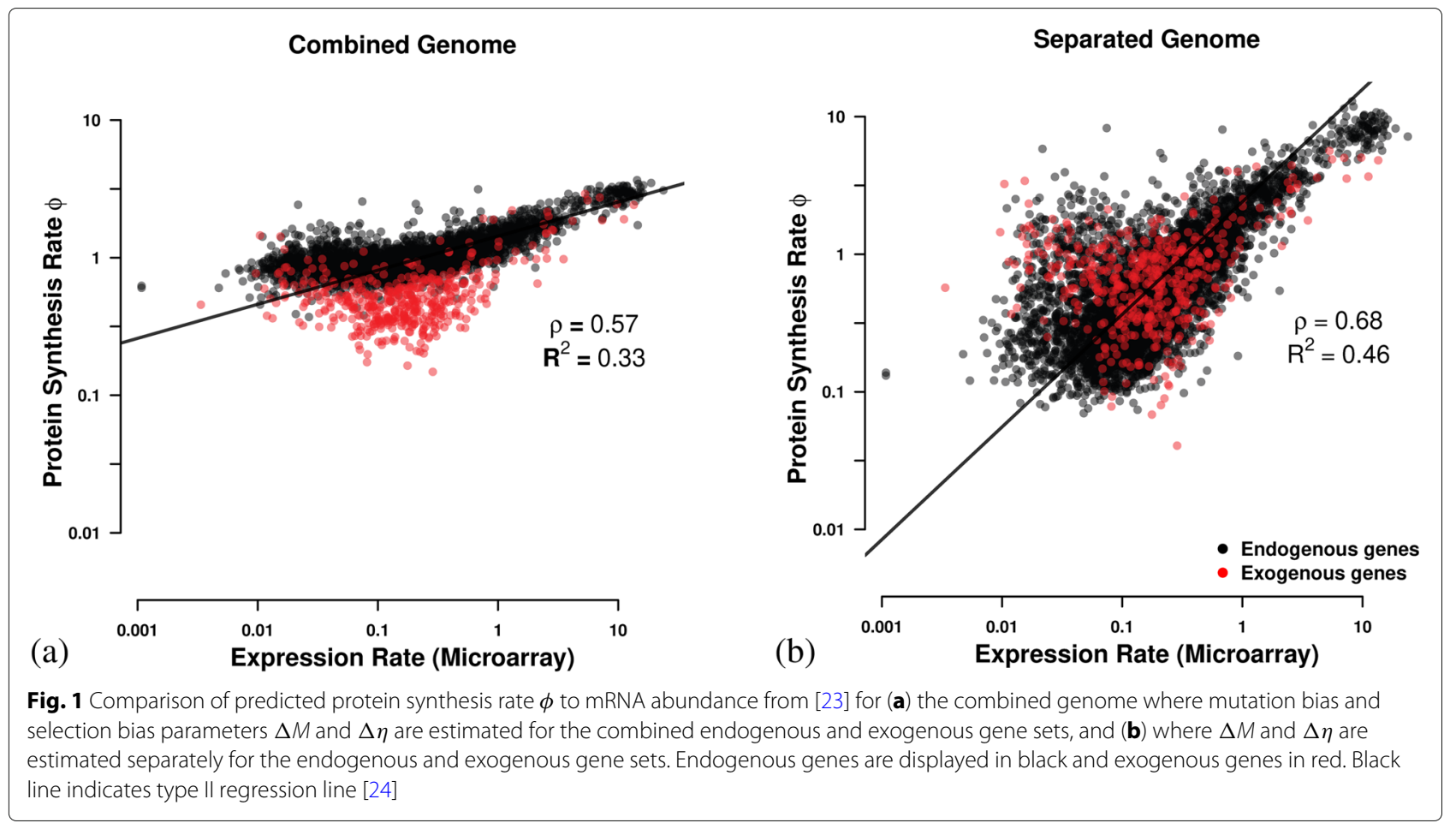

families. The exception is Leucine (Leu, L), where mutation appears to favor the codon TTG over TTA (Fig. 3, Additional file 1: Table S1). The exogenous genes display an equally consistent mutational bias towards $\mathrm{C}$ and $\mathrm{G}$ ending codons (the exception being Phe, F). In contrast to $\Delta M$, our estimates of $\Delta \eta$ for the endogenous and exogenous genes were positively correlated $(\rho=0.69)$ and showing the same sign in $\sim 53 \%$ of codons between the two selection environments (Fig. 2b).

We find that the signature of selection bias $\Delta \eta$ also differs substantially between the endogenous and exogenous gene sets. The difference in codon usage between

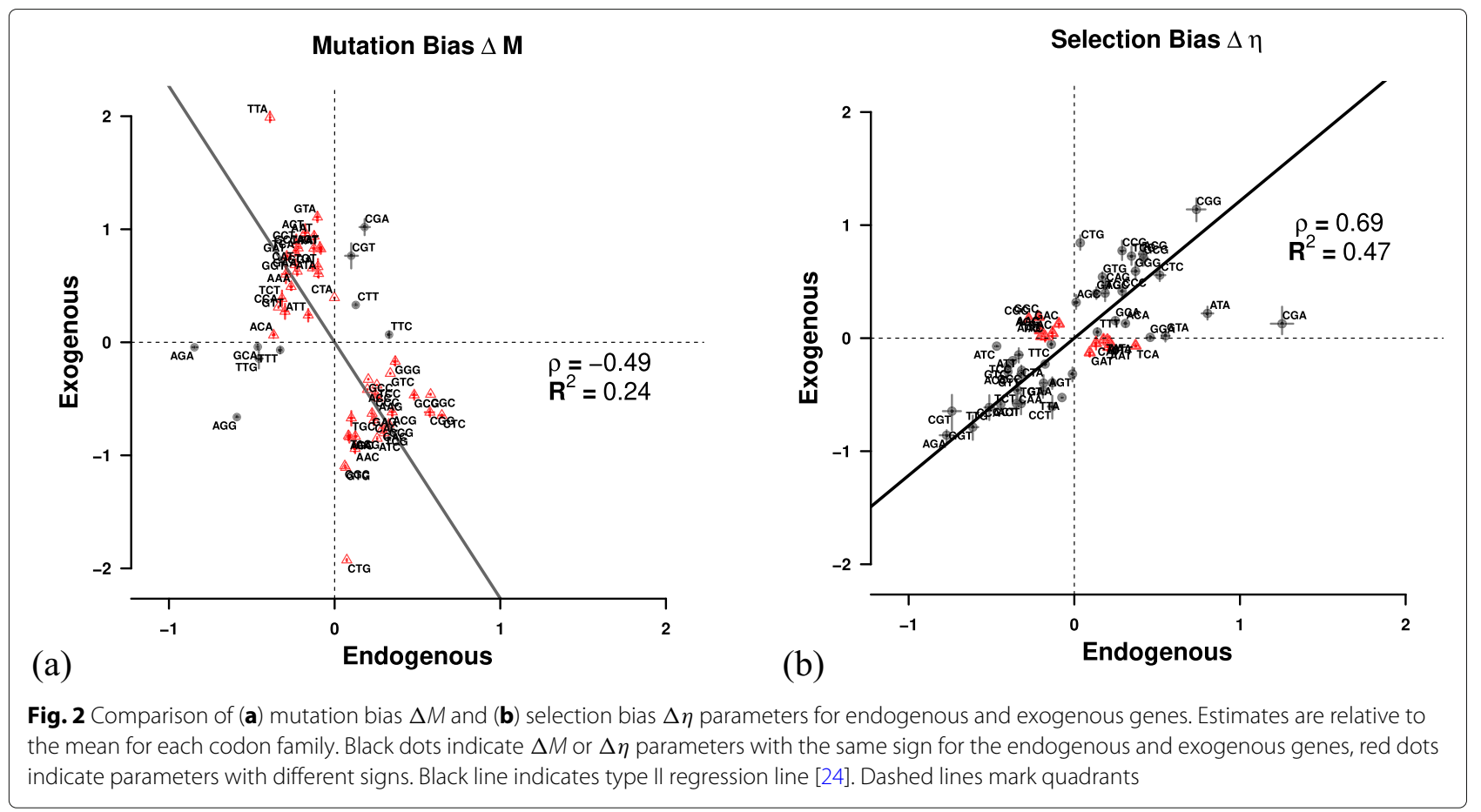




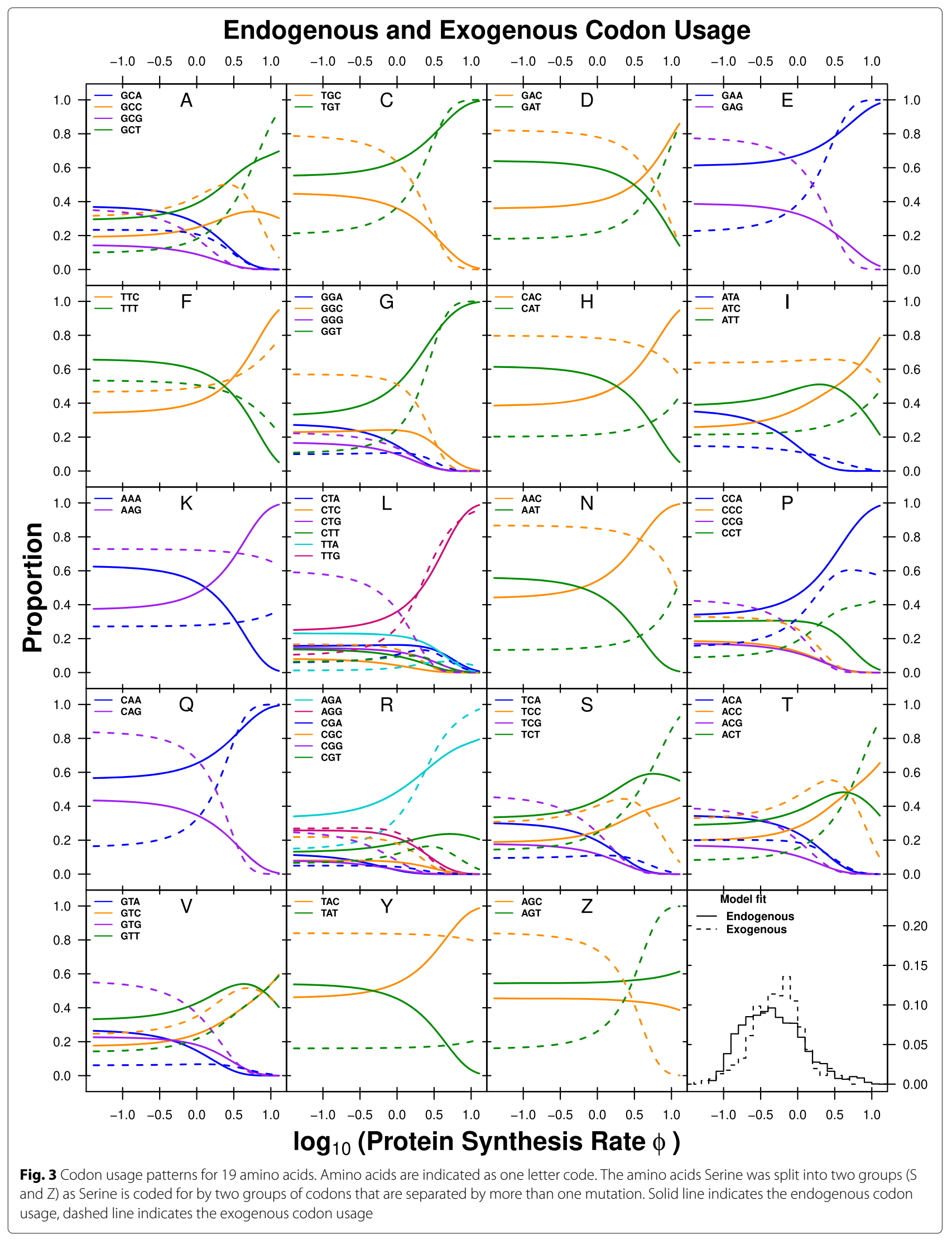


endogenous and exogenous genes is striking as the sign for $\Delta \eta$ changes, indicating a change in codon preference for some amino acids. As a result, our estimates of the optimal codon differ in nine cases between endogenous and exogenous genes (Fig. 3, Additional file 1: Table S2). For example, the usage of the Asparagine (Asn, $\mathrm{N})$ codon AAC is increased in highly expressed endogenous genes but the same codon is depleted in highly expressed exogenous genes. For Aspartic acid (Asp, D), the combined genome shows the same codon preference in highly expressed genes as the exogenous gene set. Generally, fits to the complete $L$. kluyveri genome reveal that the relatively small exogenous gene set ( $\sim 10 \%$ of genes) has a disproportionate effect on the model fit (Additional file 1: Figures S3, S4a,b).

Of the nine cases in which the endogenous and exogenous genes show differences in the selectively most favored codon five cases (Asp, D; His, H; Lys, K; Asn, N; and Pro, P) the endogenous genes favor the codon with the most abundant tRNA. For the remaining four cases (Ile, I; Ser, S; Thr, T; and Val, V), there are no tRNA genes for the wobble free cognate codon encoded in the $L$. kluyveri genome. However, the codon preference of these four amino acids in the exogenous genes matches the most abundant tRNA encoded in the L. kluyveri genome. In contrast to $\Delta M$, our estimates of selection bias $\Delta \eta$ for the endogenous and exogenous genes are positively correlated $\left(\rho=0.69, p=9.76 \times 10^{-10}\right)$ and show the same sign in $\sim 53 \%$ of the cases (Fig. 2, Additional file 1: Figure S5a).

This striking difference in codon usage was noted previously. For example, using RSCU [5], GAA (coding for Glu, E) was identified as the optimal synonymous codon in the whole genome and GAG as the optimal codon in the exogenous genes [15]. Our results, however, indicate that GAA is the optimal codon in both, endogenous and exogenous genes, and that the high RSCU in the exogenous genes of GAG is driven by mutation bias (Additional file 1: Table S1 and S2). Similar effects are observed for other amino acids.

The effect of the small exogenous gene set on the fit to the complete L. kluyveri genome is smaller for our estimates of selection bias $\Delta \eta$ than $\Delta M$, but still large (Additional file 1: Figure S5b). We find that the complete L. kluyveri genome is estimated to share the selectively preferred codon with the exogenous genes in $\sim 60 \%$ of codon families that show dissimilarity between endogenous and exogenous genes. We also find that the complete L. kluyveri genome fit shares mutationally preferred codons with the exogenous genes in $\sim 78 \%$ of the 19 codon families showing a difference in mutational codon preference between the endogenous and exogenous genes. In two cases, Isoleucine (Ile, I) and Arginine (Arg, R), the strong dissimilarity in mutation preference results in an estimated codon preference in the complete L. kluyveri genome that differs from both the endogenous, and the exogenous genes. These results clearly show that it is important to recognize the difference in endogenous and exogenous genes and treat these genes as separate sets to avoid the inference of incorrect synonymous codon preferences and better predict protein synthesis.

\section{Can codon usage help determine the source of the exogenous genes}

Since the origin of the exogenous genes is currently unknown, we explored if the information on codon usage extracted from the exogenous genes can be used to identify a potential source lineage. We combined our estimates of mutation bias $\Delta M$ and selection bias $\Delta \eta$ with synteny information and searched for potential source lineages of the introgressed exogenous region. We used $\Delta M$ to identify candidate lineages as the endogenous and exogenous genes show greater dissimilarity in mutation bias than in selection bias. We examined 332 budding yeasts [21] and, identified the ten lineages with the highest correlation to the exogenous $\Delta M$ parameters as potential source lineages (Fig. 4, Table 2). Two of the ten candidate lineages utilize the alternative yeast nuclear code (NCBI codon table 12). In this case, the codon CTG codes for Serine instead of Leucine. We therefore excluded the Leucine codon family from our comparison of codon families; however, there was no need to exclude Serine as CTG is not a one step neighbor of the remaining Serine codons. A

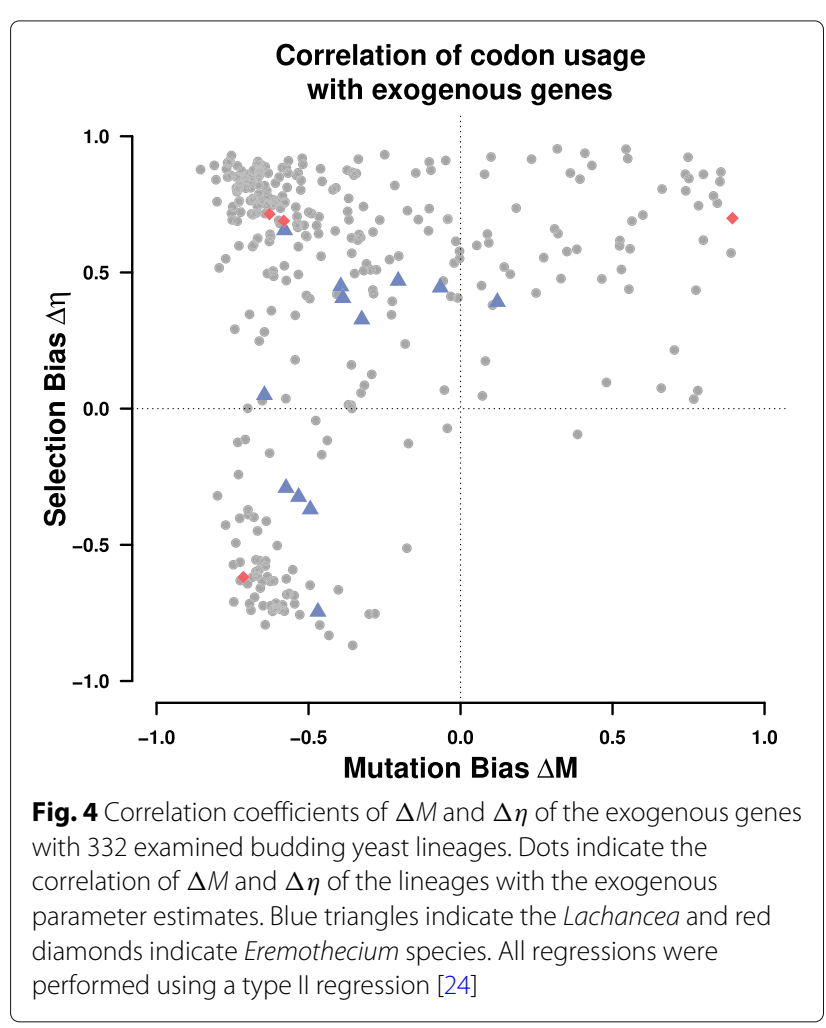


Table 2 Budding yeast lineages showing similarity in codon usage with the exogenous genes

\begin{tabular}{|c|c|c|c|c|c|}
\hline Species & $\rho_{\Delta M}$ & $\rho_{\Delta \eta}$ & GC content & Synteny \% & Distance [Mya] \\
\hline Eremothecium gossypii & 0.89 & 0.70 & 51.7 & 75 & 211.0847 \\
\hline Danielozyma ontarioensis & 0.75 & 0.92 & 46.6 & 3 & 470.1043 \\
\hline Metschnikowia shivogae & 0.86 & 0.87 & 49.8 & 0 & 470.1043 \\
\hline Babjeviella inositovora & 0.83 & 0.78 & 48.1 & 0 & 470.1044 \\
\hline Ogataea zsoltii & 0.75 & 0.85 & 47.7 & 0 & 470.1042 \\
\hline Metschnikowia hawaiiensis & 0.80 & 0.86 & 44.4 & 0 & 470.1042 \\
\hline Candida succiphila & 0.85 & 0.83 & 40.9 & 0 & 470.1042 \\
\hline Middelhovenomyces tepae & 0.80 & 0.62 & 40.8 & 0 & 651.9618 \\
\hline Candida albicans* & 0.84 & 0.75 & 33.7 & 0 & 470.1043 \\
\hline Candida dubliniensis* & 0.78 & 0.75 & 33.1 & 0 & 470.1043 \\
\hline
\end{tabular}

*Lineages use the alternative yeast nuclear code

$\rho_{\Delta M}$ and $\rho_{\Delta \eta}$ represent the Pearson correlation coefficient for exogenous $\Delta M$ and $\Delta \eta$ with the indicated species', respectively. GC content is the average GC content of the whole genome. Synteny is the percentage of the exogenous genes found in the listed lineage. Only one lineage (E. gossypii) shows a similar GC content > 50\%

mutation between CTG and the remaining Serine codons would require two mutations with one of them being non-synonymous, which would violate the weak mutation assumption of ROC SEMPPR.

The endogenous L. kluyveri genome exhibits codon usage very similar to most (77\%) yeast lineages examined, indicating that most of the examined yeasts share a similar codon usage (Additional file 1: Figure S6). Only 17\% of all examined yeast show a positive correlation in both, $\Delta M$ and $\Delta \eta$ with the exogenous genes, whereas the vast majority of lineages $(\sim 83 \%)$ show a negative correlation for $\Delta M$, only $21 \%$ show a negative correlation for $\Delta \eta$.

Comparing synteny between the exogenous genes, which are restricted to the left arm of chromosome $C$, and the candidate yeast species we find that E. gossypii is the only species that displays high synteny (Table 2). Furthermore, the synteny relationship between the exogenous region and other yeasts appears to be limited to Saccharomycetaceae clade. Given these results, we conclude that, of the 332 examined yeast lineages the E. gossypii lineage is the most likely source of the introgressed exogenous genes. Previous studies which studied the exogenous genes and chromosome recombination in the Lachancea clade concluded that the exogenous region originated from within the Lachancea clade, from an unknown or potentially extinct lineage [15-17]. While it is not possible for us to dispute this hypothesis, our results provide a novel hypothesis about the origin of the exogenous genes.

To further test the plausibility of E. gossypii as potential source linage, we identified 127 genes in our dataset [21] with homologous genes in E. gossypii and other Lachancea and used IQTree [25] to infer the phylogenetic relationship of the exogenous genes. Our results show that at least $\sim 45 \%$ of exogenous genes (57/127) are more closely related to E. gossypii than to other Lachancea (Additional file 1: Figure S7). Interestingly, our results also indicate that codon usage does not necessarily correlate with phylogenetic distance (Table 2).

\section{Estimating introgression age}

If we assume that the exogenous genes originated from the E. gossypii lineage, we can estimate the age of the introgression based on our estimates of mutation bias $\Delta M$. We modeled the change in codon frequency over time as exponential decay, and estimated the age of the introgression assuming that E. gossypii still represents the mutation bias of its ancestral source lineage at the time of the introgression and a constant mutation rate. We infer the age of the introgression to be on the order of $6.2 \pm 1.2 \times 10^{8}$ generations. Assuming L. kluyveri experiences between one and eight generations per day, we estimate the introgression to have occurred between 212,000 to $1,700,000$ years ago. Our estimate places the time of the introgression earlier than the previous estimate of 19,000 - 150,000 years by [16].

Using our model of exponential decay model, we also estimated the persistence of the signal of the exogenous cellular environment. We predict that the $\Delta M$ signal of the source cellular environment will have decayed to be within one percent of the L. kluyveri environment in $\sim$ $5.4 \pm 0.2 \times 10^{9}$ generations, or between $1,800,000$ and $15,000,000$ years. Together, these results indicate that the mutation signature of the exogenous genes will persist for a very long time.

\section{Estimating selection against codon mismatch of the exogenous genes}

We define the selection against inefficient codon usage as the difference between the fitness on the log scale of an expected, replaced endogenous gene and the exogenous gene, $s \propto \phi \Delta \eta$ due to the mismatch in codon usage parameters (See Methods for details). As the intro- 
gression occurred before the diversification of L. kluyveri and has fixed throughout all populations [16], we can not observe the original endogenous sequences that have been replaced by the introgression. Overall, we predict that a small number of low expression genes $(\phi<1)$ were weakly exapted at the time of the introgression (Fig. 5a). Thus, they appear to provide a small fitness advantage due to the accordance of exogenous mutation bias with endogenous selection bias (compare Additional file 1: Figures S3 and S4). High expression genes $(\phi>1)$ are predicted to have faced the largest selection against their mismatched codon usage in the novel cellular environment. In order to account for differences in the efficacy of selection on codon usage either due to the cost of pausing, differences in the effective population size, or the decline in fitness with every ATP wasted between the donor lineage and L. kluyveri we added a linear scaling factor $\kappa$ to scale our estimates of $\Delta \eta$ between the donor lineage and L. kluyveri and searched for the value that minimized the cost of the introgression, thus giving us the best case scenario (See Methods for details).

Using our estimates of $\Delta M$ and $\Delta \eta$ from the endogenous genes and assuming the current exogenous amino acid composition of genes is representative of the replaced endogenous genes, we estimate the strength of selection against the exogenous genes at the time of introgression (Fig. 5a) and currently (Fig. 5b). Estimates of selection bias for the exogenous genes show that, while well correlated with the endogenous genes, only nine amino acids share the same selectively preferred codon. Exogenous genes are, therefore, expected to represent a significant reduction in fitness for L. kluyveri due to mismatch in codon usage. Since $\Delta \eta$ is proportional to the difference in fit- ness between the wild type and a mutant, we can use our estimates of $\Delta \eta$ to approximate the selection against the exogenous genes $\Delta s[10,26]$. We estimate that the selection against all exogenous genes due to mismatched codon usage to have been $\Delta s \approx-0.0008$ at the time of the introgression and $\approx-0.0003$ today. This reduction in $\Delta s$ is primarily due to adaptive changes to the codon usage of the most highly expressed, introgressed genes (Fig. 5a \& Additional file 1: Figure S8). Based on the selection against the codon mismatch at the time of the introgression and assuming an effective population size $N_{e}$ on the order of $10^{7}$ [27], we estimate a fixation probability of $(1-\exp [-\Delta s]) /\left(1-\exp \left[-2 \Delta s N_{e}\right]\right) \approx 10^{-6952}[26]$ for the exogenous genes. Clearly, the possibility of fixation under this simple scenario is effectively zero. In order for the exogenous genes to have reached fixation one or more exogenous loci must have provided a selective advantage not considered in this study (See Discussion).

\section{Discussion}

In order to study the evolutionary effects of the large scale introgression of the left arm of chromosome $\mathrm{C}$, we used ROC SEMPPR, a mechanistic model of ribosome movement along an mRNA. The usage of a mechanistic model rooted in population genetics allows us generate more nuanced quantitative parameter estimates and separate the effects of mutation and selection on the evolution of codon usage. This allowed us to calculate the selection against the introgression, and provides $E$. gossypii as a potential source lineage of the introgression which was previously not considered. Our parameter estimates indicate that the L. kluyveri genome contains distinct signatures of mutation and selection bias from both

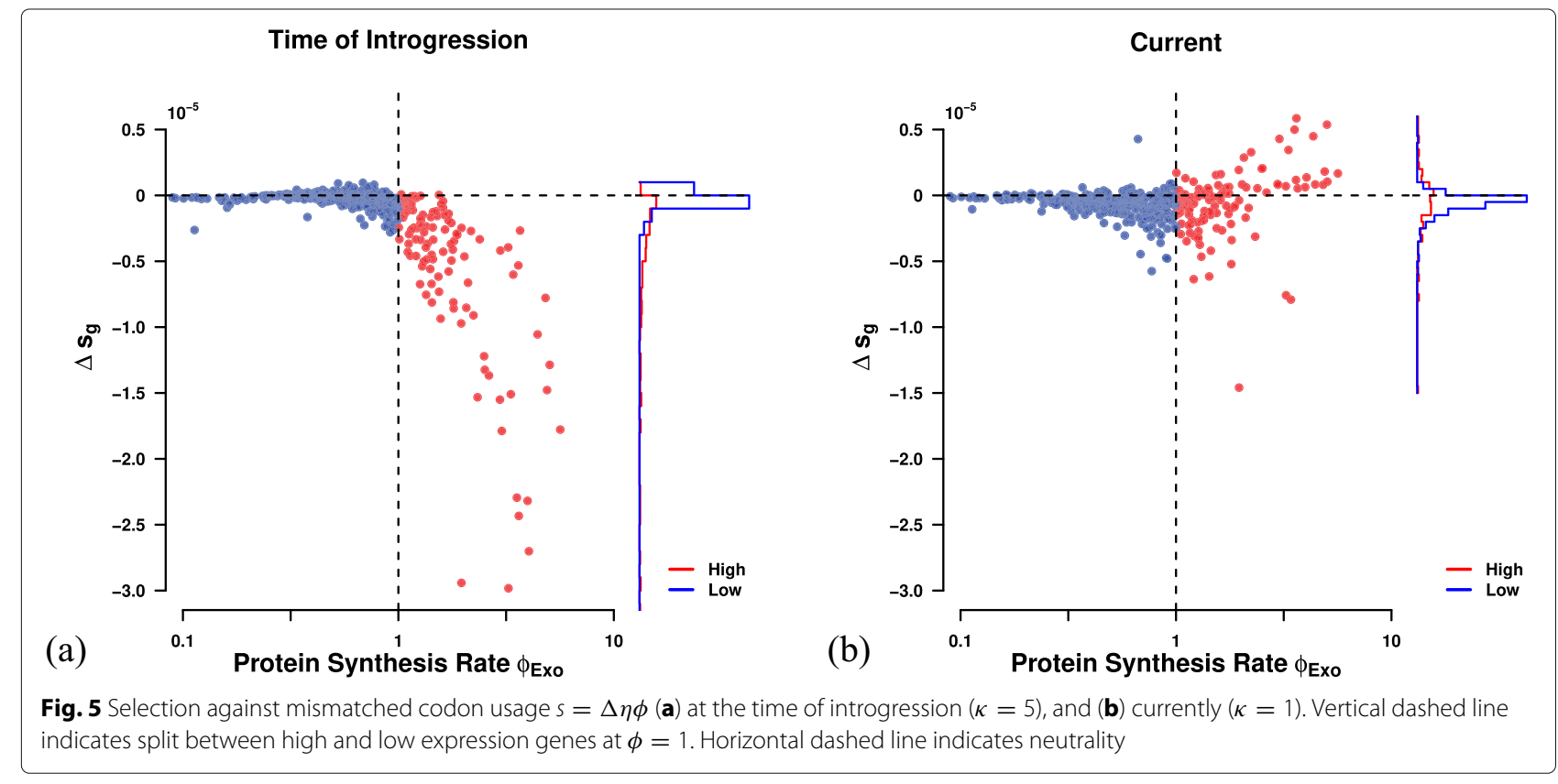


an endogenous and exogenous cellular environment. By fitting ROC SEMPPR separately to L. kluyveri's endogenous and exogenous sets of genes we generate a quantitative description of their signatures of mutation bias and natural selection for efficient protein translation.

In contrast to other methods such as RSCU, CAI, or tAI, ROC SEMPPR does not rely on external information such as gene expression or tRNA gene copy number $[5,19]$. Instead, ROC SEMPPR allows for the estimation of protein synthesis rate $\phi$ and separates the effects of mutation and selection on codon usage. In addition, [20] showed that approaches like CAI are sensitive to amino acid composition, another property that distinguishes the endogenous and exogenous genes [15].

Previous work by [15] showed an increased bias towards $\mathrm{GC}$ rich codons in the exogenous genes but our results provide more nuanced insights by separating the effects of mutation bias and selection. We are able to show that the difference in GC content between endogenous and exogenous genes is mostly due to differences in mutation bias as 95\% of exogenous codon families show a strong mutation bias towards GC ending codons (Additional file 1: Table S1). However, the exogenous genes show a selective preference for AT ending codons for $90 \%$ of codon families (Additional file 1: Table 2). Acknowledging the increased mutation bias towards GC ending codons and the difference in strength of selection between endogenous and exogenous genes by separating them also improves our estimates of protein synthesis rate $\phi$ by $42 \%$ relative to the full genome estimate $\left(R^{2}=0.46, p=0\right.$ vs. $0.32, p=0$, respectively).

Previous studies showed that nucleotide composition can be strongly affected by biased gene conversion, which, in turn would affect codon usage. Biased gene conversion is thought to act similar to directional selection, typically favoring the fixation of G/C alleles [28, 29]. Further, Harrison and Charlesworth [30] suggested that biased gene conversion affects codon usage in S. cerevisiae. ROC SEMPPR, however, does not explicitly account for biased gene conversion. If biased gene conversion is independent of gene expression, as in the case of DNA repair, it will be absorbed in our estimates of $\Delta M$. If instead biased gene conversion forms hotspots, and thus becomes gene specific, it will affect our estimates of protein synthesis $\phi$. This might be the case at recombination hotspots. Recombination, however, is very low in the introgressed region (discussed below) [15, 18]. The low recombination rate also indicates that the GC content had to be high before the introgression occurred.

The estimates of mutation and selection bias parameters, $\Delta M$ and $\Delta \eta$, are obtained under an equilibrium assumption. Given that the introgression is still adapting to its new environment, this assumption is clearly violated. However, the adaptation of the exogenous genes progresses very slowly as a quasi-static process as shown in this work as well as [16]. Therefore, the genome can be assumed to maintain an internal equilibrium at any given time. We see empirical evidence for this behavior in our ability to predict gene expression and to correctly identify the low expression genes (Fig. 1b).

Despite the violation of the equilibrium assumption, the mutation and selection bias parameters $\Delta M$ and $\Delta \eta$ of the introgressed exogenous genes contain information, albeit decaying, about its previous cellular environment. We selected the top ten lineages with the highest similarity in $\Delta M$ to see if our parameters estimates would allow us to identify a potential source lineage. The synteny relationship of these lineages with the exogenous genes was calculated as a point of comparison as it provides orthogonal information to our parameter estimates. Synteny with the exogenous genes is limited to the Saccharomycetaceae clade, excluding all of the potential source lineages identified using codon usage but E. gossypii (Table 2). Interestingly, this also showed that similarity in codon usage does not correlate with phylogenetic distance.

Previous work indicated that the donor lineage of the exogenous genes has to be a, potentially unknown, Lachancea lineage [15-18]. These previous results, however, are based on species rather than gene trees, ignoring the differential adaptation rate to their novel cellular environment between genes or do not consider lineages outside of the Lachancea clade. Considering the similarity in selection bias (Fig. 2b) and our calculation of selection on the exogenous genes (Fig. 5b), both of which are free of any assumption about the origin of the exogenous genes, a species tree estimated from the exogenous genes will be biased towards the Lachancea clade. Estimating individual gene trees rather than relying on a species tree provided further evidence that the exogenous genes could originate from a lineage that does not belong to the Lachancea clade. As we highlighted in this study, relatively small sets of genes with a signal of a foreign cellular environment can significantly bias the outcome of a study. The same holds true for phylogenetic inferences [31], and as we showed the signal of the original endogenous cellular environment that shaped CUB is at different stages of decay in high and low expression genes (Additional file 1: Figure S8). In summary, our work does not dispute an unknown Lachancea as possible origin, but provides an alternative hypothesis based on the codon usage of the exogenous genes, phylogenetic analysis, and synteny.

In terms of understanding the spread of the introgression, we calculated the expected selective cost of codon mismatch between the L. kluyveri and E. gossypii lineages. Under our working hypothesis, the majority of the introgressed would have imposed a selective cost due to codon mismatch. Nevertheless, $\sim 30 \%$ of low expression exogenous genes $(\phi<1)$ appeared to be exapted at the 
time of the introgression. This exaptation is due to the mutation bias in the endogenous genes matching the selection bias in the exogenous genes for GC ending codons. Our estimate of the selective cost of codon mismatch on the order of -0.0008 . While this selective cost may not seem very large, assuming $L$. kluyveri had a large $N_{e}$, the fixation probability of the introgression is the astronomically small value of $\approx 10^{-6952} \approx 0$. While this estimate heavily depends on the working hypothesis that the exogenous genes originated from the E. gossypii lineage, we can also calculate the hypothetical fixation probability if the current exogenous genes would introgress into L. kluyveri. Our estimate of the current selective cost of the mismatch of codon usage is on the order of -0.0003 . The fixation probability of the current exogenous genes would still be astronomically small $\approx 10^{-2609} \approx 0$ These results are in accordance with previous work, highlighting the necessity of codon usage compatibility between endogenous and transferred exogenous genes [32, 33]. Thus, the basic scenario of an introgression between two yeast species with large $N_{e}$ and where the introgression solely imposes a selective cost due to codon mismatch is clearly too simplistic.

One or more loci with a combined selective advantage on the order of 0.0008 or greater would have made the introgression change from disadvantageous to effectively neutral or advantageous. While this scenario seems plausible, it raises the question as to why recombination events did not limit the introgression to only the adaptive loci. A potential answer is the low recombination rate between the endogenous and exogenous regions $[15,18]$. Estimates of the recombination rate as measured by crossovers (COs) for L. kluyveri are almost four times lower than for S. cerevisae and about half that of Schizosaccharomyces pombe $(\approx 1.6 \mathrm{COs} / \mathrm{Mb} /$ meiosis,$\approx 6 \mathrm{COs} / \mathrm{Mb} /$ meiosis, $\approx 3 \mathrm{COs} / \mathrm{Mb} /$ meiosis) with no observed crossovers in the introgressed region [18], and no observed transposable elements [15]. This is presumably due to the dissimilarity in GC content and/or a lower than average sequence homology between the exogenous region and the one it replaced. A population bottleneck reducing the $N_{e}$ of the L. kluyveri lineage around the time of the introgression could also help explain the spread of the introgression. Compatible with these explanation is the possibility of several advantageous loci distributed across the exogenous region drove a rapid selective sweep and/or the population through a bottleneck speciation process.

Assuming E. gossypii as potential source lineage of the exogenous region, we illustrated how information on codon usage can be used to infer the time since the introgression occurred using our estimates of mutation bias $\Delta M$. The $\Delta M$ estimates are well suited for this task as they are free of the influence of selection and unbiased by $N_{e}$ and other scaling terms, which is in contrast to our estimates of $\Delta \eta$ [10]. Our estimated age of the introgression of $6.2 \pm 1.2 \times 10^{8}$ generations is $\sim 10$ times longer than a previous minimum estimate by [16] of $5.6 \times 10^{7}$ generations, which was based on the effective population recombination rate and the population mutation parameter [34]. Furthermore, these estimates assume that the current E. gossypii and L. kluyveri cellular environment reflect their ancestral states at the time of the introgression. Thus, if the ancestral mutation environments were more similar (dissimilar) at the time of the introgression then our result is an overestimate (underestimate).

Further, the presented work provides a template to explore the evolution of codon usage. This applies not only to species who experienced an introgression but is more generally applicable to any species.

\section{Conclusion}

Overall, our results show the usefulness of the separation of mutation bias and selection bias and the importance of recognizing the presence of multiple cellular environments in the study of codon usage. We also illustrate how a mechanistic model like ROC SEMPPR and the quantitative estimates it provides can be used for more sophisticated hypothesis testing in the future. In contrast to other approaches used to study codon usage like CAI [5] or tAI [19], ROC SEMPPR incorporates the effects of mutation bias and amino acid composition explicitly [20]. We highlight potential issues when estimating codon preferences, as estimates can be biased by the signature of a second, historical cellular environment. In addition, we show how quantitative estimates of mutation bias and selection relative to drift can be obtained from codon data and used to infer the fitness cost of an introgression as well as its history and potential future.

\section{Methods}

\section{Separating endogenous and exogenous genes}

A GC-rich region was identified by [15] in the L. kluyveri genome extending from position 1 to 989,693 of chromosome $C$. This region was later identified as an introgression by [16]. We obtained the L. kluyveri genome from SGD Project http://sgd-archive.yeastgenome.org/ sequence/fungi/ (on 09-27-2014) and the annotation for L. kluyveri NRRL Y-12651 (assembly ASM14922v1) from NCBI (on 12-09-2014). We assigned 457 genes located on chromosome $\mathrm{C}$ with a location within the $\sim 1 \mathrm{Mb}$ window to the exogenous gene set. All other 4864 genes of the $L$. kluyveri genome were assigned to the exogenous genes.

\section{Model fitting with ROC SEMPPR}

ROC SEMPPR was fitted to each genome using AnaCoDa (0.1.1) [22] and R (3.4.1) [35]. ROC SEMPPR was run from 10 different starting values for at least 250,000 iterations 
and thinned to keep every 50th iteration. After manual inspection to verify that the MCMC had converged, parameter posterior means, log posterior probability and log likelihood were estimated from the last 500 samples (last $10 \%$ of samples).

\section{Model selection}

The marginal likelihood of the combined and separated model fits was calculated using a generalized harmonic mean estimator [36]. A variance scaling of 1.1 was used to scale the important density of the estimator. Using the estimated marginal likelihoods, we calculated the Bayes factor to assess model performance. Increases in the variance scaling increase the estimated Bayes factor, therefore we report a conservative Bayes factor bases on a small variance scaling Additional file 1: Figure S2.

\section{Comparing codon specific parameter estimates and selecting candidate lineages}

As the choice of reference codon can reorganize codon families coding for an amino acid relative to each other, all parameter estimates were interpreted relative to the mean for each codon family.

$$
\begin{aligned}
& \Delta M_{i}=\Delta M_{i, 1}-\overline{\Delta M_{i}} \\
& \Delta \eta_{i}=\Delta \eta_{i, 1}-\overline{\Delta \eta_{i}}
\end{aligned}
$$

Comparison of codon specific parameters ( $\Delta M$ and $\Delta \eta=$ $\left.2 N_{e} q\left(\eta_{i}-\eta_{j}\right)\right)$ was performed using the function lmodel2 in the R package lmodel2 (1.7.3) [37] and R version 3.4.1 [35]. The parameter $\Delta \eta$ can be interpreted as the difference in fitness between codon $i$ and $j$ for the average gene with $\phi=1$ scaled by the effective population size $N_{e}$, and the selective cost of an ATP $q[4,10]$. Type II regression was performed with re-centered parameter estimates, accounting for noise in dependent and independent variable [24].

Due to the greater dissimilarity of the $\Delta M$ estimates between the endogenous and exogenous genes, and the slower decay rate of mutation bias, we decided to focus on our estimates of mutation bias to identify potential source lineages. The top ten lineages with the highest similarity in $\Delta M$ to the exogenous genes were selected as potential candidates (Fig. 2).

\section{Phylogenetic analysis}

Using the dataset from [21], we first identified 129 alignments for exogenous genes that further contained homologous genes for E. gossypii, and at least one other Lachancea species. We excluded all species from the alignments that do not belong to the Saccharomycetaceae clade. IQTree [25] was used to identify the best fitting model for each gene and to estimate the individual gene trees. Each gene tree was rooted using either
Saccharomyces cerevisiae, Saccharomyces uvarum, Saccharomyces eubayanus as outgroup. We calculated the most recent common ancestor (MRCA) of L. kluyveri and E. gossypii as well as the MRCA of $L$. kluyveri and the remaining Lachancea. The distance between the MRCA and the root was used to asses which pairs (L. kluyveri and E. gossypii, or L. kluyveri and other Lachancea) have a more recent common ancestor.

\section{Synteny comparison}

We obtained complete genome sequences for all 10 candidate lineages (Table 2) from NCBI (on: 02-05-2017). Genomes were aligned and checked for synteny using SyMAP (4.2) with default settings [38, 39]. We assess synteny as percentage coverage of the exogenous gene region.

\section{Estimating age of introgression}

We modeled the change in codon frequency over time using an exponential model for all two codon amino acids. While our approach is equivalent to [40], we want to explicitly state the relationship between the change in codon frequency $c_{1}$ as a function of mutation bias $\Delta M$ as

$$
\frac{d c_{1}}{d t}=-\mu_{1,2} c_{1}-\mu_{2,1}\left(1-c_{1}\right)
$$

where $\mu_{i, j}$ is the rate at which codon $i$ mutates to codon $j$ and $c_{1}$ is the frequency of the reference codon. Initial codon frequencies $c_{1}(0)$ for each codon family were taken from our mutation parameter estimates for E. gossypii where $c_{1}(0)=\exp \left[\Delta M_{\text {gos }}\right] /\left(1+\exp \left[\Delta M_{\text {gos }}\right]\right)$. Our estimates of $\Delta M_{\text {endo }}$ can be used to calculate the steady state of Eq. 3 were $\frac{d c_{1}}{d t}=0$ to obtain the equality

$$
\frac{\mu_{2,1}}{\mu_{1,2}+\mu_{2,1}}=\frac{1}{1+\exp \left[\Delta M_{\text {endo }}\right]}
$$

Solving for $\mu_{1,2}$ gives us $\mu_{1,2}=\Delta M_{\text {endo }} \exp \left[\mu_{2,1}\right]$ which allows us to rewrite and solve Eq. 3 as

$$
c_{1}(t)=\frac{1+\exp [-X](K-1)}{1+\Delta M_{\text {endo }}}
$$

where $X=\left(1+\Delta M_{\text {endo }}\right) \mu_{2,1} t$ and $K=c_{1}(0)\left(1+\Delta M_{\text {endo }}\right)$. Equation 5 was solved with a mutation rate $\mu_{2,1}$ of $3.8 \times$ $10^{-10}$ per nucleotide per generation [41]. Current codon frequencies for each codon family where taken from our estimates of $\Delta M$ from the exogenous genes. Mathematica (11.3) [42] was used to calculate the time $t_{\text {intro }}$ it takes for the initial codon frequencies $c_{1}(0)$ for each codon family to equal the current exogenous codon frequencies. The same equation was used to determine the time $t_{\text {decay }}$ at which the signal of the exogenous cellular environment has decayed to within $1 \%$ of the endogenous environment. 


\section{Estimating selection against codon mismatch}

In order to estimate the selection against codon mismatch, we had to make three key assumptions. First, we assumed that the current exogenous amino acid sequence of a gene is representative of its ancestral state and the replaced endogenous gene it replaced. Second, we assume that the currently observed cellular environment of $E$. gossypii reflects the cellular environment that the exogenous genes experienced before transfer to L. kluyveri. Lastly, we assume that the difference in the efficacy of selection between the cellular environments due to differences in either effective population size $N_{e}$ or the selective cost of an ATP $q$ of the source lineage and L. kluyveri can be expressed as a scaling constant and that protein synthesis rate $\phi$ has not changed between the replaced endogenous and the introgressed exogenous genes. Using estimates for $N_{e}=1.36 \times 10^{7}$ [27] for Saccharomyces paradoxus we scale our estimates of $\Delta \eta$ which explicitly contains the effective population size $N_{e}$ [10] and define $\Delta \eta^{\prime}=\frac{\Delta \eta}{N_{e}}$.

All of our genome parameter estimations are scaled by lineage specific effects such as $N_{e}$, the average, absolute gene expression level, and/or the proportionate fitness value of an ATP. In order to account for these genome specific differences in scaling, we scale the difference in the efficacy of selection on codon usage between the donor lineage and L. kluyveri using a linear scaling factor $\kappa$. As $\Delta \eta$ is defined as $\Delta \eta=2 N_{e} q\left(\eta_{i}-\eta_{j}\right)$, we cannot distinguish if $\kappa$ is a scaling on protein synthesis rate $\phi$, effective population size $N_{e}$, or the selective cost of an ATP $q$ $[4,10]$. We calculated the selection against each genes codon mismatch assuming additive fitness effects as

$$
s_{g}=\sum_{i=1}^{L_{g}}-\kappa \phi_{g} \Delta \eta_{i}^{\prime}
$$

where $s_{g}$ is the overall strength of selection for translational efficiency on gene, $g$ in the exogenous gene set, $\kappa$ is a constant, scaling the efficacy of selection between the endogenous and exogenous cellular environments, $L_{g}$ is length of the protein in codons, $\phi_{g}$ is the estimated protein synthesis rate of the gene in the endogenous environment, and $\Delta \eta_{i}^{\prime}$, is the $\Delta \eta^{\prime}$ for the codon at position $i$. As stated previously, our $\Delta \eta$ are relative to the mean of the codon family. We find that the selection against the introgressed genes is minimized at $\kappa \sim 5$ (Additional file 1: Figure S9b). Thus, we expect a five fold difference in the efficacy of selection between $L$. kluyveri and $E$. gossypii, due to differences in either protein synthesis rate $\phi$, effective population size $N_{e}$, and/or the selective cost of an ATP $q$. Therefore, we set $\kappa=1$ if we calculate the $s_{g}$ for the endogenous and the current exogenous genes, and $\kappa=5$ for $s_{g}$ for selection calculations at the time of introgression.
However, since we are unable to observe codon sequences of the replaced endogenous genes and for the exogenous genes at the time of introgression, instead of summing over the sequence, we calculate the expected codon count $E\left[n_{g, i}\right]$ for codon $i$ in gene $g$ simply as the probability of observing codon $i$ multiplied by the number of times the corresponding amino acids is observed in gene $g$, yielding:

$$
\begin{aligned}
E\left[n_{g, i}\right] & =P\left(c_{i} \mid \Delta M, \Delta \eta, \phi\right) \times m_{a_{i}} \\
& =\frac{\exp \left[-\Delta M_{i}-\Delta \eta_{i} \phi_{g}\right]}{\sum_{j}^{C} \exp \left[-\Delta M_{j}-\Delta \eta_{j} \phi_{g}\right]} \times m_{a_{i}}
\end{aligned}
$$

where $m_{a_{i}}$ is the number of occurrences of amino acid $a$ that codon $i$ codes for. Thus replacing the summation over the sequence length $L_{g}$ in Eq. (6) by a summation over the codon set $C$ and calculating $s_{g}$ as

$$
s_{g}=\sum_{i=1}^{C}-\kappa \phi_{g} \Delta \eta_{i}^{\prime} E\left[n_{g, i}\right]
$$

We report the selection due to mismatched codon usage of the introgression as $\Delta s_{g}=s_{\text {intro, } g}-s_{\text {endo, } g}$ where $s_{\text {intro, } g}$ is the selection against an introgressed gene $g$ either at the time of the introgression or presently.

\section{Supplementary information}

Supplementary information accompanies this paper at https://doi.org/10.1186/s12862-020-01649-w.

Additional file 1: Supplementary Material. Supporting Materials for Unlocking a signal of introgression from codons in Lachancea kluveri using a mutation-selection model by Landerer et al.

\section{Abbreviations}

AIC: Akaike information criterion; CAl: Codon adaptation index; CUB: Codon usage bias; ROC SEMPPR: Ribosome Overhead Cost Stochastic Evolutionary Model of Protein Production Rate; RSCU: Relative synonymous codon usage; tAl: tRNA adaptation index

\section{Acknowledgments}

The authors would like to thank Alexander Cope for helpful criticisms and suggestions for this work.

\section{Authors' contributions}

$\mathrm{CL}$ and MAG initiated the study. CL collected and analyzed the data and wrote the manuscript. MAG and BCO edited the manuscript. $C L, M A G, B C O$, and $R Z$ contributed to the data analysis and acquiring of funding. All Authors approved the final manuscript.

\section{Funding}

This work was supported in part by NSF Awards MCB-1120370 (MAG and RZ), MCB-1546402 (A. Von Arnim and MAG), and DEB-1355033 (BCO, MAG, and RZ) with additional support from Department of Ecology \& Evolutionary Biology (EEB) at the University of Tennessee Knoxville (UTK) and the National Institute for Mathematical and Biological Synthesis (NIMBioS), an Institute sponsored by the National Science Foundation through NSF Award DBI-1300426. CL received support as a Graduate Student Fellow from NIMBioS with additional support from Departments of Mathematics and EEB at UTK. The funding bodies (NSF, NIMBioS, UTK) played no role in the design of the study and collection, analysis, and interpretation of the data, and the writing of the manuscript. 


\section{Availability of data and materials}

Parameter estimates generated during this study are available from the corresponding author. All remaining data generated during this study are included in this published article as figures, and tables.

\section{Ethics approval and consent to participate}

Not applicable.

\section{Consent for publication}

Not applicable.

\section{Competing interests}

The authors declare that they have no competing interests.

\section{Author details}

${ }^{1}$ Department of Ecology \& Evolutionary Biology, University of Tennessee, 37996 Knoxville, TN, USA. ${ }^{2}$ National Institute for Mathematical and Biological Synthesis, 37996 Knoxville, TN, USA. ${ }^{3}$ Max-Planck Institute of Molecular Cell Biology and Genetics, Pfotenhauerstr. 108, 01307 Dresden, Germany.

${ }^{4}$ Department of Business Analytics and Statistics, University of Tennessee, 37996 Knoxville, TN, USA.

\section{Received: 11 July 2019 Accepted: 1 July 2020}

Published online: 26 August 2020

\section{References}

1. Gouy M, Gautier C. Codon usage in bacteria: correlation with gene expressivity. Nucleic Acids Res. 1982;10:7055-74.

2. Ikemura T. Codon usage and tRNA content in unicellular and multicellular organisms. Mol Biol Evol. 1985;2:13-34.

3. Bulmer M. The selection-mutation-drift theory of synonymous codon usage. Genetics. 1990;129:897-907.

4. Gilchrist MA. Combining models of protein translation and population genetics to predict protein production rates from codon usage patterns. Mol Biol Evol. 2007;24(11):2362-72.

5. Sharp PM, Li WH. The codon adaptation index - a meassure of directional synonymous codon usage bias, and its potential applications. Nucleic Acids Res. 1987;15:1281-95.

6. Wright $F$. The 'effective number of codons' used in a gene. Gene. 1990;87:23-9.

7. M SP, Stenico M, Peden JF, Lloyd AT. Codon usage: mutational bias, translational selection, or both?. Biochem Soc Trans. 1993;21(4):835-41.

8. Shah P, Gilchrist MA. Explaining complex codon usage patterns with selection for translational efficiency, mutation bias, and genetic drift. Proc Nat Acad Sci USA. 2011;108(25):10231-6.

9. Wallace EW, Airoldi EM, Drummond DA. Estimating selection on synonymous codon usage from noisy experimental data. Mol Biol Evol. 2013;30:1438-53.

10. Gilchrist MA, Chen WC, Shah P, Landerer CL, Zaretzki R. Estimating gene expression and codon-specific translational efficiencies, mutation biases, and selection coefficients from genomic data alone. Genome Biol Evol. 2015;7:1559-79.

11. Médigue C, Rouxel T, Vigier P, Hénaut A, Danchin A. Evidence for horizontal gene transfer in Escherichia coli speciation. J Mol Biol. 1991;222(4):851-6.

12. Lawrence JG, Ochman $\mathrm{H}$. Amelioration of bacterial genomes: Rates of change and exchange. J Mol Biol. 1997;44:383-97.

13. Marcet-Houben M, Gabaldón T. Beyond the whole-genome duplication: Phylogenetic evidence for an ancient interspecies hybridization in the baker's yeast lineage. PLoS Biol. 2015;13(8):1002220.

14. Beimforde C, Feldberg K, Nylinder S, Rikkinen J, Tuovila H, Dörfelt $H_{4}$ Gube M, Jackson DJ, Reitner J, Seyfullah L, Schmidt AR. Estimating the phanerozoic history of the ascomycota lineages: combining fossil and molecular data. Mol Phylogenet Evol. 2014;78:386-98.

15. Payen C, Fischer G, Marck C, Proux C, Sherman DJ, Coppée J-Y, Johnston M, Dujon B, Neuvéglise C. Unusual composition of a yeast chromosome arm is associated with its delayed replication. Genome Res. 2009;19(10):1710-21.

16. Friedrich A, Reiser C, Fischer G, Schacherer J. Population genomics reveals chromosome-scale heterogeneous evolution in a protoploid yeast. Mol Biol Evol. 2015;32(1):184-92.
17. Vakirlis N, Sarilar V, Drillon G, Fleiss A, Agier N, Meyniel J-P, Blanpain L, Carbone A, Devillers H, Dubois K, Gillet-Markowska A, Graziani S, HuuVang N, Poirel M, Reisser C, Schott J, Schacherer J, Lafontaine I, Llorente B, Neuvéglise C, Fischer G. Reconstruction of ancestral chromosome architecture and gene repertoire reveals principles of genome evolution in a model yeast genus,. Genome Res. 2016;26(7):918-32.

18. Brion C, Legrand S, Peter J, Caradec C, Pflieger D, Hou J, Friedrich A, Llorente $B$, Schacherer J. Variation of the meiotic recombination landscape and properties over a broad evolutionary distance in yeasts. PLoS Genet. 2017;13(8):1006917.

19. dos Reis M, Savva R, Wernisch L. Solving the riddle of codon usage preferences: a test for translational selection. Nucleic Acids Res. 2004;32(17):5036-44.

20. Cope AL, Hettich RL, Gilchrist MA. Quantifying codon usage in signal peptides: Gene expression and amino acid usage explain apparent selection for inefficient codons. Biochim Biophys Acta (BBA) Biomembr. 2018;1860(12):2479-85.

21. Shen XX, Opulente DA, Kominek J, Zhou X, Steenwyk JL, Buh KV, Haase MAB, Wisecaver JH, Wang M, Doering DT, Boudouris JT, Schneider RM, Langdon QK, Ohkuma M, Endoh R, Takashima M, Manabe R, Čadež N, Libkind D, Rosa C, DeVirgilio J, Hulfachor AB, Groenewald M, Kurtzman C, Hittinger CT, Rokas A. Tempo and mode of genome evolution in the budding yeast subphylum. Cell. 2018;175(6):1533-154520.

22. Landerer C, Cope A, Zaretzki R, Gilchrist MA. AnaCoDa: analyzing codon data with bayesian mixture models. Bioinformatics. 2018;34(14):2496-8

23. Tsankov AM, Thompson DA, Socha A, Regev A, Rando OJ. The role of nucleosome positioning in the evolution of gene regulation. PLoS Biol. 2010;8(7):1000414.

24. Sokal RR, Rohlf FJ. Biometry - The principles and practice of statistics in biological. New York: WH Freeman; 1981, pp. 547-555.

25. Nguyen LT, Schmidt HA, von Haeseler A, Minh BQ. Iq-tree: A fast and effective stochastic algorithm for estimating maximum-likelihood phylogenies. Mol Biol Evol. 2015;32(1):268-74.

26. Sella G, Hirsh AE. The application of statistical physics to evolutionary biology. Proc Nat Acad Sci U S A. 2005;102:9541-6.

27. Wagner A. Energy constraints on the evolution of gene expression. Mol Biol Evol. 2005;22:1365-74.

28. Nagylaki T. Evolution of a finite population under gene conversion. Proc Nat Acad Sci U S A. 1983;80:6278-81.

29. Nagylaki T. Evolution of a large population under gene conversion. Proc Nat Acad Sci U S A. 1983;80:5941-5.

30. Harrison RJ, Charlesworth B. Biased gene conversion affects patterns of codon usage and amino acid usage in the saccharomyces sensu stricto group of yeasts. Mol Biol Evol. 2011;28(1):117-29.

31. Salichos $L$, Rokas A. Inferring ancient divergences requires genes with strong phylogenetic signals. Nature. 2013;497:327-31.

32. Medrano-Soto A, Moreno-Hagelsieb G, Vinuesa P, Christen JA, Collado-Vides J. Successful lateral transfer requires codon usage compatibility between foreign genes and recipient genomes. Mol Biol Evol. 2004;21(10):1884-94.

33. Tuller T, Girshovich Y, Sella Y, Kreimer A, Freilich S, Kupiec M, Gophna $U$, Ruppin E. Association between translation efficiency and horizontal gene transfer within microbial communities. Nucleic Acids Res. 2011;39(11):4743-55. https://doi.org/10.1093/nar/gkr054.

34. Ruderfer DM, Pratt SC, Seidl HS, Kruglyak L. Population genomic analysis of outcrossing and recombination in yeast. Nat Genet. 2006;38(9): 1077-81.

35. R Core Team. R: A Language and Environment for Statistical Computing. Vienna: R Foundation for Statistical Computing; 2013. http://www.Rproject.org/.

36. Gronau QF, Sarafoglou A, Matzke D, Ly A, Boehm U, Marsman M, Leslie DS, Forster JJ, Wagenmakers EJ, Steingroever H. A tutorial on bridge sampling. J Math Psychol. 2017;81:80-97.

37. Legendre P. Lmodel2: Model II Regression. 2018. R package version 1.7-3. https://CRAN.R-project.org/package=Imodel 2 .

38. Soderlund C, Nelson W, Shoemaker A, Paterson A. Symap A system for discovering and viewing syntenic regions of fpc maps. Genome Res. 2006;16:1159-68.

39. Soderlund C, Bomhoff M, Nelson W. Symap v3.4: a turnkey synteny system with application to plant genomes. Nucleic Acids Res. 2011;39(10):68 
40. Marais G, Charlesworth B, Wright SI. Recombination and base composition: the case of the highly self-fertilizing plant arabidopsis thaliana. Genome Biol. 2004;5:45.

41. Lang Gl, Murray AW. Estimating the per-base-pair mutation rate in the yeast Saccharomyces cerevisiae. Genetics. 2008;178(1):67-82.

42. Wolfram Research Inc. Mathematica 11. 2017. http://www.wolfram.com.

\section{Publisher's Note}

Springer Nature remains neutral with regard to jurisdictional claims in published maps and institutional affiliations.

- fast, convenient online submission

- thorough peer review by experienced researchers in your field

- rapid publication on acceptance

- support for research data, including large and complex data types

- gold Open Access which fosters wider collaboration and increased citations

- maximum visibility for your research: over $100 \mathrm{M}$ website views per year

At $B M C$, research is always in progress.

Learn more biomedcentral.com/submissions 